\title{
Role of Thermodynamics in the Shape Transformation of Embedded Metal Nanoparticles Induced by Swift Heavy-Ion Irradiation
}

\author{
M. C. Ridgway, R. Giulian, D. J. Sprouster, P. Kluth, L. L. Araujo, D. J. Llewellyn, and A. P. Byrne \\ Research School of Physics and Engineering, Australian National University, Canberra, Australia \\ F. Kremer and P. F. P. Fichtner \\ Universidade Federal do Rio Grande do Sul, Porto Alegre, Brazil \\ G. Rizza \\ Ecole Polytechnique, Laboratoire des Solides Irradies, CEA-IRAMIS-CNRS, Palaiseau, France
}

H. Amekura

National Institute for Materials Science, Tsukuba, Japan

M. Toulemonde

Laboratoire CIMAP-GANIL, Caen, France

(Received 4 November 2010; published 2 March 2011)

\begin{abstract}
Swift heavy-ion irradiation of elemental metal nanoparticles (NPs) embedded in amorphous $\mathrm{SiO}_{2}$ induces a spherical to rodlike shape transformation with the direction of NP elongation aligned to that of the incident ion. Large, once-spherical NPs become progressively more rodlike while small NPs below a critical diameter do not elongate but dissolve in the matrix. We examine this shape transformation for ten metals under a common irradiation condition to achieve mechanistic insight into the transformation process. Subtle differences are apparent including the saturation of the elongated NP width at a minimum sustainable, metal-specific value. Elongated NPs of lesser width are unstable and subject to vaporization. Furthermore, we demonstrate the elongation process is governed by the formation of a molten ion-track in amorphous $\mathrm{SiO}_{2}$ such that upon saturation the elongated NP width never exceeds the molten ion-track diameter.
\end{abstract}

DOI: 10.1103/PhysRevLett.106.095505

Ion-solid interactions during swift heavy-ion irradiation (SHII) are dominated by inelastic processes in the form of electron excitation and ionization while, in contrast, the influence of elastic processes such as ballistic displacements is negligible. Macroscopically, amorphous $\mathrm{SiO}_{2}$ $\left(a-\mathrm{SiO}_{2}\right)$ undergoes a volume-conserving anisotropic deformation when subjected to SHII such that thin freestanding layers contract and expand, respectively, in directions parallel and perpendicular to that of the incident ion [1]. The viscoelastic model $[2,3]$, based on a transient thermal effect, successfully explains this so-called ion hammering. Microscopically, energy is deposited along the ion path, from incident ion to matrix electrons, and is then dissipated within a narrow cylinder of material surrounding the ion path. The heat flow in both the electron and lattice subsystems is well described as functions of time and radial distance by the inelastic thermal spike $(i-\mathrm{TS})$ model $[4,5]$. When the temperature of the lattice exceeds that required for melting, the material along the ion path is molten and upon quenching an ion track is formed. Recently, we measured the molten ion-track diameter in $\mathrm{a}-\mathrm{SiO}_{2}$ as a function of electronic stopping power [6]. The ion-track radial density distribution consisted of an under-dense core and over-dense shell (relative to
PACS numbers: 61.80.Jh, 81.40.Wx, 61.46.Hk, 65.80.-g

unirradiated material), the formation of which was attributed to a quenched-in pressure wave emanating from the ion-track center [6].

Elemental metal nanoparticles (NPs) embedded in $a-\mathrm{SiO}_{2}$ and subjected to SHII can undergo an intriguing shape transformation where once-spherical NPs become progressively more rodlike with the direction of elongation aligned along that of the incident ion. This phenomenon has been reported for several metals under a wide range of SHII conditions, with Refs. [7-17] citing selected examples. Freestanding metallic NPs irradiated under comparable conditions do not change shape, demonstrating the embedding $a-\mathrm{SiO}_{2}$ matrix must have a role in the shape transformation process $[8,17]$. An unambiguous identification of the atomistic mechanism underlying the transformation remains lacking but a metal NP melt-and-flow process, the latter potentially as a means of relieving in-plane stress within the matrix $[8,14,17]$, is certainly plausible. For this report, we examine the SHII-induced elongation of ten embedded, elemental metal NP systems using a common irradiation condition to enable the identification of subtle, metal-specific differences and demonstrate that the thermodynamic properties of both the matrix and metal are intrinsic to the shape transformation process. 
Elemental metal nanoparticles of $\mathrm{Co}, \mathrm{Ni}, \mathrm{Cu}, \mathrm{Zn}, \mathrm{Ag}$, $\mathrm{Sn}, \mathrm{Pt}, \mathrm{Au}, \mathrm{Pb}$, and $\mathrm{Bi}$ were formed over a depth of $\sim 0.2-1.5 \mu \mathrm{m}$ in an $a-\mathrm{SiO}_{2}$ layer of thickness $2 \mu \mathrm{m}$ on a crystalline $\mathrm{Si}$ substrate by ion implantation and thermal annealing. The processing conditions were necessarily metal specific but in general yielded a metal concentration of 2-10 at. \% after implantation and a broad NP size distribution after annealing. All NPs were approximately spherical in shape, independent of the metal species. Samples were then irradiated at room temperature and normal incidence with $185 \mathrm{MeV} \mathrm{Au}{ }^{+13}$ ions where the electronic stopping power in $a-\mathrm{SiO}_{2}$ is approximately $17 \mathrm{keV} / \mathrm{nm}[18]$.

The shape transformation was characterized with crosssectional transmission electron microscopy (XTEM) measurements of the NP minor and major dimensions ( $d_{\text {min }}$ and $d_{\text {maj }}$, respectively) or, equivalently, the NP width and length. For statistical reliability $\sim 500$ NPs per sample were measured. As an example, Fig. 1 shows XTEM images of $\mathrm{Ni}, \mathrm{Cu}$ and $\mathrm{Bi}$ NPs following SHII where the once-spherical metal NPs are elongated in the incident-ion direction as expected. The spherical-to-rodlike shape transformation has not been previously reported for these three metals and thus such results further demonstrate that the SHII-induced shape transformation is operative across a broad compositional and crystallographic range of metal NPs embedded in $a-\mathrm{SiO}_{2}$.

Figure 2 shows results for $d_{\min }$ as a function of $d_{\text {maj }}$ for Co NPs following SHII where individual values of $d_{\text {min }}$ represent an average of $d_{\text {min }}$ for all NPs of a given $d_{\text {maj }} \pm$ $1 \mathrm{~nm}$. For unirradiated NPs, the slope of 1 is indicative of their spherical shape. Upon irradiation, elongation is manifested as $d_{\text {maj }}>d_{\text {min }}$ though clearly only for NPs above a certain size. For Co NPs under the given irradiation conditions, NPs of diameter less than $\sim 7 \mathrm{~nm}$ are not elongated. Though such behavior was common to all the metals, the numerical value of this threshold diameter $\left(d_{\text {thres }}\right)$ was metal specific, varying from $\sim 6 \mathrm{~nm}$ for Pt to
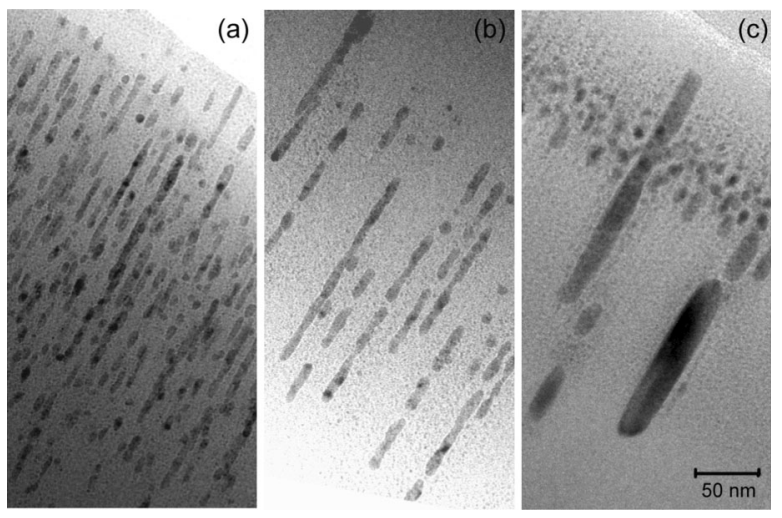

FIG. 1. XTEM images of (a) Ni, (b) $\mathrm{Cu}$ and (c) Bi NPs following SHII. The incident-ion direction was from the top right to bottom left of the image.
10-11 nm for lower melting-point metals. As discussed below, we suggest the origin of $d_{\text {thres }}$ stems from the instability of small NPs under ion irradiation.

For an incident ion intersecting a spherical metal NP of radius $\frac{d_{\min }}{2}\left(=\frac{d_{\text {maj }}}{2}\right)$, the deposited energy density per atom $\left(E_{\text {dep(sphere) }}\right)$ within the NP is

$$
E_{\text {dep(sphere })}=\frac{\varepsilon l}{\frac{4}{3} \pi\left(\frac{d_{\min }}{2}\right)^{2}\left(\frac{d_{\text {maj }}}{2}\right) N},
$$

where $\varepsilon$ and $l$ are the ion electronic energy loss in the NP and path length in the NP, respectively, and $N$ is the atomic density. (While we have assumed all the energy is deposited along the ion path, following Ref. [19] one can show that in bulk material $>85 \%$ of the energy is deposited within $5 \mathrm{~nm}$ of the ion-track center for the ten metals under consideration. Furthermore, the potential barrier at the metal $/ \mathrm{SiO}_{2}$ interface confines electrons of energy $<4-5 \mathrm{eV}$ to the metal and thus to a good approximation the energy deposited in the NP remains within the NP.) If the ion intersects the NP at a distance $B$ from the NP axis, $l$ is given by

$$
l=2 \frac{d_{\mathrm{maj}}}{d_{\mathrm{min}}} \sqrt{\left(\frac{d_{\mathrm{min}}}{2}\right)^{2}-B^{2}}
$$

such that

$$
E_{\mathrm{dep}(\text { sphere })}=\frac{12 \varepsilon}{\pi d_{\min }^{3} N} \sqrt{\left(\frac{d_{\min }}{2}\right)^{2}-B^{2}} .
$$

When the fraction of $E_{\text {dep(sphere) }}$ transferred to the metal lattice exceeds that required for vaporization, the NP is vaporized and dissolved in the matrix. Clearly $E_{\text {dep(sphere) }}$ scales as approximately $\left(1 / d_{\min }^{2}\right)$ and, for any given metal, smaller NPs are thus more susceptible to vaporization than

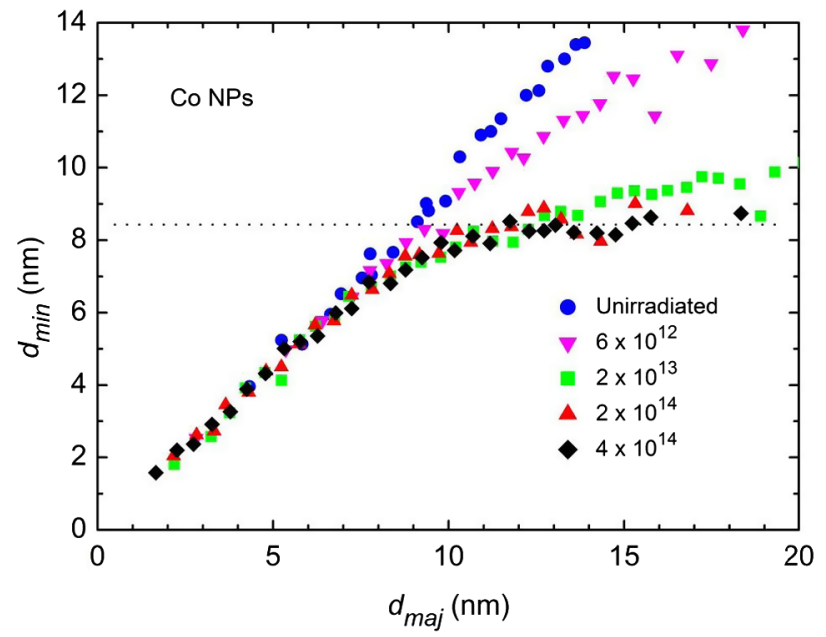

FIG. 2 (color online). Elongated NP width $\left(d_{\text {min }}\right)$ as a function of elongated NP length $\left(d_{\text {maj }}\right)$ following SHII of Co NPs. Irradiation fluences $\left(/ \mathrm{cm}^{2}\right)$ are listed. 
their larger counterparts. As dissolved metal and matrix atoms cool, metal atoms may remain dispersed in the matrix, form new clusters and/or be absorbed by morestable, larger NPs. Our x-ray absorption near-edge spectroscopy (XANES) and small-angle $x$-ray scattering (SAXS) measurements showed the fraction of metal atoms dissolved in the matrix increased as a function of SHII fluence and, furthermore, the rate of dissolution increased as the mean NP diameter decreased $[12,16]$.

For spherical NPs of diameter $>d_{\text {thres }}$, Fig. 2 demonstrates the shape transformation proceeds gradually as a function of irradiation fluence with the slope beyond the minimum size threshold progressively approaching zero. The latter indicates that all elongated NPs eventually attain a common width independent of their length which suggests that this $d_{\min (\text { saturation) }}$ is a characteristic parameter for NPs elongated by SHII. The $d_{\min (\text { saturation) }}$ values were determined for the ten different metals and, to better relate this data to the thermodynamic properties of both the metal and matrix, results are plotted in Fig. 3 as a function of the energy density per atom required to vaporize $\left(E_{\text {vap }}\right)$ the bulk metal. We calculated this parameter using

$E_{\text {vap }}=\frac{C_{s}\left(T_{\text {melt }}-T_{\text {irrad }}\right)+L_{\text {melt }}+C_{s}\left(T_{\text {vap }}-T_{\text {melt }}\right)+L_{\text {vap }}}{N\left(1.6 \times 10^{-19}\right)}$,

where $C_{s}$ is the specific heat, $T_{\text {melt }}, T_{\text {irrad }}$ and $T_{\text {vap }}$ are the melting, irradiation and vaporization temperatures, respectively, and $L_{\text {melt }}$ and $L_{\text {vap }}$ are the latent heats of melting and vaporization, respectively. For such calculations, we used the thermodynamic parameters of the bulk metals. Finitesize effects in elemental metal NPs can induce changes in the structural, vibrational and thermodynamic properties relative to bulk material including differences in bond

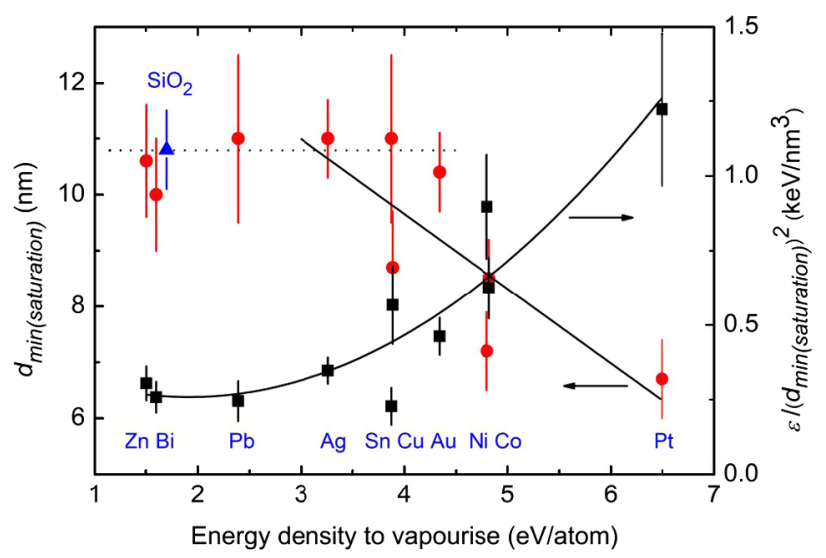

FIG. 3 (color online). Saturated NP width $\left(d_{\text {min(saturation })}\right)$ and $\varepsilon /\left(d_{\min (\text { saturation })}\right)^{2}$ as a function of the energy density per atom required for vaporization. The horizontal line is the molten iontrack diameter in $a-\mathrm{SiO}_{2}$ and the associated experimental uncertainty is indicated by the error bars on the triangular symbol. length [20-23], Einstein temperature $[23,24]$ and melting temperature [25]. However, these effects are typically significant only for NPs of diameter considerably less than those used in this report and, as a consequence, any error introduced through our use of bulk values was considered minimal.

With reference to Fig. 3, two distinct trends are apparent: $d_{\min (\text { saturation) }}$ is effectively constant at low $E_{\text {vap }}$ then decreases approximately linearly at higher values. We consider these two dependencies separately, first addressing the near-constant behavior at low $E_{\text {vap }}$.

We have previously measured the diameter of the molten ion-track induced in $a-\mathrm{SiO}_{2}$ by SHII for the irradiation conditions used in this report [6]. The horizontal line in Fig. 3 represents this value and the triangular symbol labeled $\mathrm{SiO}_{2}$ shows the associated experimental error. Note how $d_{\min (s a t u r a t i o n)}$ never exceeds the molten ion-track diameter. We previously speculated that these two parameters were correlated $[12,14,16]$ and the extensive measurements presented herein confirm that the maximum width of an embedded and elongated NP, irradiated to a fluence sufficient for saturation, is restricted to the diameter of the molten ion track in $a-\mathrm{SiO}_{2}$. We suggest this is compelling evidence that metal NP melt and flow, the latter within the molten ion track formed in the matrix, is intrinsic to the shape transformation of metal NPs in $a-\mathrm{SiO}_{2}$ induced by SHII.

To now interpret the approximately linear decrease in $d_{\text {min(saturation) }}$ apparent at high $E_{\text {vap }}$, consider an incident ion intersecting an elongated metal NP, assumed a prolate spheroid with dimensions $d_{\text {min }}$ and $d_{\text {maj }}$ and volume $\left(4 / 3\left[\pi\left(\frac{d_{\min }}{2}\right)^{2}\left(\frac{d_{\text {maj }}}{2}\right)\right]\right)$. The deposited energy density per atom $E_{\text {dep(prolatespheroid) }}=E_{\text {dep(sphere) }}$ and thus also scales as approximately $\left(1 / d_{\min }^{2}\right)$ independent of the NP length $d_{\text {maj }}$ [see Eq. (3)]. Figure 2 demonstrated the spherical-torodlike shape transformation progresses gradually as a function of fluence consistent with hundreds of ion-track overlaps. As the NPs increase in length and decrease in width, the deposited energy density per atom necessarily increases. We contend $d_{\min (\text { saturation })}$ is the minimum sustainable width of an elongated NP under ion irradiation and speculate that for lesser widths, the fraction of $E_{\text {dep(prolatespheroid) }}$ transferred to the metal lattice exceeds that required for vaporization and the elongated NP is vaporized just as we observed for small spherical NPs of diameter less than $d_{\text {thres. }}$. In Fig. 3, we also plot $\left(\varepsilon / d_{\min (\text { saturation })}^{2}\right)$ as a function of $E_{\mathrm{vap}}$. Clearly metals such as Pt that require larger values of energy density per atom to vaporize are able to sustain larger values of deposited energy density per atom and smaller values of $d_{\min (\text { saturation) }}$. Note also that our XANES [16] and SAXS [12] measurements for $\mathrm{Co}$ and $\mathrm{Pt}$, respectively, suggest that all NPs, both spherical and rodlike, eventually dissolve in the matrix for sufficiently high ion fluences. 
Clearly, the thermodynamics of both matrix and metal have a role in the shape transformation of metal NPs induced by SHII; while the maximum width of an elongated NP is limited by the diameter of the molten ion track in the matrix, the minimum width is governed by the energy density per atom required for vaporization of the metal. This requires the conversion from the liquid (molten metal) to vapour (gaseous metal) state with the latter necessarily confined in the matrix by the ion track and potentially accommodated by the under-dense core. For our irradiation conditions, the ion track core is also vaporized [6]. The $\sim 1.5 \mathrm{~nm}$ radius within which the vapor phase exists is insufficient to account for our observed $d_{\min (\text { saturation) }}$ in contrast to the $\sim 5 \mathrm{~nm}$ radius of the molten phase. We also considered other thermodynamic properties of the metal including the closely related cohesive energy. While we observed the same trend to that shown in Fig. 3, we believe our argument based on the energy density per atom required for vaporization is best suited to physical interpretation and enables us to better identify the mechanisms of melting, flow, elongation and vaporization operative during the spherical-to-rodlike shape transformation of embedded metal NPs.

In summary, we have investigated the SHII-induced shape transformation in a variety of elemental metal NPs embedded in $a-\mathrm{SiO}_{2}$. Spherical NPs below a threshold diameter did not elongate while those of greater size progressively transformed to a rodlike shape of fixed width independent of length. Utilizing a common irradiation condition enabled us to identify metal-specific differences in both the spherical NP threshold diameter and elongated NP width and relate such differences to the width of the molten ion track in $a-\mathrm{SiO}_{2}$ and the energy density per atom required for vaporization of the metal NP. Spherical NPs below the threshold diameter for elongation and elongated NPs below the saturation width are unstable under SHII and are vaporized as a result of the interaction with an incident ion. While this defines the minimum sustainable width of an elongated NP, the maximum is restricted to the width of the ion track in $a-\mathrm{SiO}_{2}$ within which the molten metal flows.
We thank the Australian Research Council for financial support, the Australian National Fabrication Facility for equipment access and the staff of the Heavy-Ion Accelerator Facility of the Australian National University for technical assistance.

[1] A. Benyagoub, S. Klaumunzer, and M. Toulemonde, Nucl. Instrum. Methods Phys. Res., Sect. B 146, 449 (1998).

[2] H. Trinkaus and A. I. Ryazanov, Phys. Rev. Lett. 74, 5072 (1995).

[3] T. van Dillen et al., Phys. Rev. B 71, 024103 (2005).

[4] M. Toulemonde, C. Dufour, and E. Paumier, Phys. Rev. B 46, 14362 (1992) and references therein.

[5] M. Toulemonde et al., K. Dan. Vidensk. Selsk. Mat. Fys. Medd. 52, 263 (2006).

[6] P. Kluth et al., Phys. Rev. Lett. 101, 175503 (2008).

[7] C. D’Orleans et al., Phys. Rev. B 67, 220101 (2003).

[8] S. Roorda et al., Adv. Mater. 16, 235 (2004).

[9] A. Oliver et al., Phys. Rev. B 74, 245425 (2006).

[10] J. J. Penninkhof et al., Nucl. Instrum. Methods Phys. Res., Sect. B 242, 523 (2006).

[11] Y. K. Mishra et al., Appl. Phys. Lett. 91, 063103 (2007).

[12] R. Giulian et al., Phys. Rev. B 78, 125413 (2008).

[13] K. Awazu et al., Phys. Rev. B 78, 054102 (2008).

[14] P. Kluth et al., Appl. Phys. Lett. 94, 113107 (2009).

[15] E. A. Dawi et al., J. Appl. Phys. 105, 074305 (2009).

[16] M. C. Ridgway et al., Nucl. Instrum. Methods Phys. Res., Sect. B 267, 931 (2009).

[17] R. Giulian et al., Phys. Rev. B 82, 113410 (2010).

[18] J. F. Ziegler, J. P. Biersack, and U. Littmark, The Stopping and Range of Ions in Solids (Pergamon, New York, 1985).

[19] M. P. R. Waligorski, R. N. Hann, and R. Katz, Nucl. Tracks Rad. Meas. 11, 309 (1986).

[20] P. Kluth et al., Appl. Phys. Lett. 85, 3561(2004).

[21] B. Johannessen et al., J. Appl. Phys. 98, 024307 (2005).

[22] R. Giulian et al., J. Appl. Phys. 105, 044303(2009).

[23] D. J. Sprouster et al., J. Appl. Phys. 107, 014313 (2010).

[24] R. Giulian et al., J. Phys. Condens. Matter 21, 155302 (2009).

[25] K. Koga, T. Ikeshoji, and K. Sugawara, Phys. Rev. Lett. 92, 115507 (2004). 